\title{
A PROBLEM OF BOSĂK CONCERNING THE GRAPHS OF SEMIGROUPS
}

\author{
Y.-F. LIN
}

Let $S$ be a semigroup, $S$ the set of all proper subsemigroups of $S$. By the graph $G(S)$ of $S$, we mean the nondirected graph whose set of vertices is $\mathrm{S}$, in which vertices $A$ and $B$ are adjacent (that is, are joined by an edge) if and only if $A \neq B$ and $A \cap B \neq \varnothing$. Recall that a graph is connected provided there is a path between every pair of its vertices. In his paper [1], Bosák proved: If $S$ is a nondenumerable semigroup or a periodic semigroup with more than two elements, then its graph $G(\delta)$ is connected. Bosák then raised the following open problem.

Bosák's Problem [1, p. 122]:1 Does there exist a semigroup with more than two elements whose graph is disconnected?

The purpose of this article is to answer this problem, in the negative, by proving the following theorem.

THEOREM. Every semigroup with more than two elements has a connected graph.

We remark that the particular semigroup $\{0,1\}$ with the multiplication of integers has a disconnected graph, and this turns out to be the only one such, as the theorem asserted. The graph of the trivial semigroup, which consists of one element alone, is trivially connected. Thus, henceforth we need only to consider a semigroup with more than two elements.

We first prove two special cases which will be used in the proof of the general case. A unital [resp. left unital] semigroup is a semigroup with a unit (=identity) [resp. left unit (=left identity)].

LEMMA 1. If $S$ is a unital semigroup with more than two elements, then its graph $G(\delta)$ is connected.

Proof. Suppose, to obtain a contradiction, that there exists a unital semigroup $S$ with more than two elements such that its graph $G(\delta)$ is not connected. Then, there exist two disjoint nonempty subsets $\delta_{1}$ and $S_{2}$ of $\delta$ such that $S_{1} \cup S_{2}=\delta$, and no semigroup in $\delta_{1}$ has an element in common with any semigroup in $\delta_{2}$. Let 1 denote the unit of $S$; there is no loss of generality in assuming the semigroup

Received by the editors June 1, 1968.

${ }^{1}$ I am indebted to Anthony $\mathrm{C}$. Shershin for calling my attention to this problem. 
$\{1\} \in \delta_{1}$. Let $S_{2}$ be an arbitrary semigroup in $\delta_{2}$, and let $x \in S_{2}$. Then since the subsemigroup $\left\{1, x, x^{2}, x^{2}, \cdots\right\}$ intersects both $\{1\} \in S_{1}$ and $S_{2} \in S_{3}$, by the disconnectedness of $G(\delta)$ we must have $S$ $=\left\{1, x, x^{2}, x^{2}, \cdots\right\}$, because $\left\{1, x, x^{2}, x^{2}, \cdots\right\}$ cannot be a proper subsemigroup.

Case $1 . S=\left\{1, x, x^{2}, x^{2}, \cdots\right\}$ is finite.

In this case, $S$ is a periodic semigroup with more than two elements, and hence by Theorem 1 of Bosák [1], its graph $G(S)$ is connected.

Case 2. $S=\left\{1, x, x^{2}, x^{2}, \cdots\right\}$ is infinite.

In this case, for any two positive integers $m$ and $n, x^{m} \neq x^{n}$ if and only if $m \neq n\left[2\right.$, p. 19]. Consequently, $\left\{1, x^{2}, x^{4}, x^{6}, \cdots\right\}$ is a proper subsemigroup of $S$ which intersects both $\{1\} \in \mathcal{S}_{1}$ and $S_{2} \in \mathcal{S}_{2}$. This contradiction completes the proof of the lemma.

LEMMA 2. If $S$ is a left unital semigroup with more than two elements, then its graph $G(\$)$ is connected.

Proof. Let $e$ be a left unit of $S$, then there are the following two cases.

Case 1. $S=S e=\{x e: x \in S\}$.

In this case, $e$ is also a right unit of $S$, and hence $S$ has $e$ as the unit. Consequently, by Lemma 1 , the graph $G(\delta)$ is connected.

Case 2. $S \neq$ Se.

Suppose that the graph $G(\delta)$ is not connected, then $\delta$ is a disjoint union of its nonempty subsets $S_{1}$ and $S_{2}$ such that no semigroups in $\delta_{1}$ intersect any semigroup in $\delta_{2}$. Under this case, $S e$ is a proper subsemigroup of $S$ and hence $S e$ must belong to either $S_{1}$ or $S_{2}$, say $S_{1}$. Take any subsemigroup $S_{\mathbf{2}}$ from $S_{\mathbf{2}}$, and take any element $x$ from $S_{\mathbf{2}}$. The set $S_{0}=\left\{e, x, x^{2}, x^{3}, \cdots\right\} \cup\left\{x e, x^{2} e, x^{3} e, \cdots\right\}$, which is easily seen to be a subsemigroup of $S$, intersects both $S e \in S_{1}$ and $S_{\mathbf{2}} \in \mathcal{S}_{\mathbf{2}}$. Consequently, $S_{0}$ cannot be a proper subsemigroup of $S$. Thus, $S$ $=\left\{e, x, x^{2}, x^{3}, \cdots\right\} \cup\left\{x e, x^{2} e, x^{3} e, \cdots\right\}$. Let $S_{3}=\left\{e, x^{2}, x^{4}, x^{6}, \cdots\right\}$ $\cup\left\{x^{2} e, x^{4} e, x^{6} e, \cdots\right\}$, which is readily seen to be a subsemigroup of $S$. As assumed in the beginning of this proof the graph $G(S)$ is disconnected, it follows from Theorem 1 of [1] that $S$ must have infinitely many elements. Now, if $x \in\left\{e, x^{2}, x^{4}, x^{6}, \cdots\right\}$, then $S_{z}$ is finite and hence it is a member of $s$. If $x \notin\left\{e, x^{3}, x^{4}, x^{6}, \cdots\right\}$, then $S_{3}$ is a member of $S$ also. On the other hand, since $S_{3}$ contains the element $x^{2} e$ of $S e \in \delta_{1}$ and also the element $x^{2}$ of $S_{2} \in S_{2}, S_{3}$ can not belong to $\delta_{1} \cup S_{2}=\delta$. The contradiction proves that the graph $G(\delta)$ is connected.

We are now ready to prove our theorem stated earlier. 
Proof of Theorem. Suppose, to obtain a contradiction, that there exists a semigroup $S$ with more than two elements such that its graph $G(\delta)$ is disconnected. Then, there exist two vertices (proper subsemigroups) $A$ and $B$ such that there is no path connecting $A$ and $B$. Choose two elements $a \in A$ and $b \in B$; and keep them fixed throughout the proof. Since $a S$ and $S b$ are subsemigroups of $S$ such that $a^{2} \in A$ $\cap a S, a b \in a S \cap S b$ and $b^{2} \in S b \cap B$, we must have $a S=S$ or $S b=S$. For, otherwise, there would be a path in $G(S)$ connecting $A$ and $B$. Assume $a S=S$ (the case $S b=S$ may be handled similarly), then $A_{1}=\{x: x \in S, x S=S\}$ forms a subsemigroup containing $a$.

Case $1 . A_{1} \neq S$; that is, $A_{1} \in \delta$.

From the choice of $a, b$ in $S$ and the assumption $A_{1} \neq S$, it follows that $b \notin A_{1}$. Since $a^{2} \in A \cap S a, b a \in b S \cap S a, b^{2} \in b S \cap B$ and $b S \neq S$, we must have $S a=S$. Otherwise, there would be a path in $G(S)$ connecting $A$ and $B . S a=S$ implies that there exists an element $e \in S$ such that $e a=a$. And $a S=S$ implies that for every $x$ in $S$ there exists an element $y$ in $S$ such that $x=a y$. Whence, $e x=e(a y)=(e a) y=a y=x$. Thus, $S$ is left unital and therefore, by Lemma 2, the graph $G(S)$ is connected.

Case 2. $A_{1}=S$; that is, $x S=S$ for all $x \in S$.

We divide this case into two subcases:

Subcase 2.1. There exists some $c \in S$ such that $S c=S$.

Using the same arguments employed in Case 1 above, $c S=S=S c$ implies that $S$ is left unital, and hence that the graph $G(S)$ is connected.

Subcase 2.2. $S x \neq S$ for all $x \in S$.

For any positive integers $m$ and $n, S a^{m}$ and $S b^{n}$ are two proper subsemigroups of $S$ such that $a^{m+1} \in A \cap S a^{m}$ and $b^{n+1} \in B \cap S b^{n}$. Hence $S a^{m} \cap S b^{n}=\varnothing$; for otherwise, there would be a path connecting $A$ and $B$, contradicting the assumption about $A$ and $B$. We next observe that $S=S a^{m} \cup S b^{n}$ for all positive integers $m$ and $n$. For if otherwise, $a^{m+1} \in A \cap\left(S a^{m} \cup S b^{n}\right)$ and $b^{n+1} \in\left(S a^{m} \cup S b^{n}\right) \cap B$ imply that there is a path between $A$ and $B$, which again is a contradiction.

From the above paragraph, we now conclude that $S a=S a^{m}$ and $S b=S b^{n}$ for all positive integers $m$ and $n$. Let us denote $T=S a$, then $a T=a(S a)=(a S) a=S a=T$ and $T a=(S a) a=S a^{2}=S a=T$.

These imply that there exists an element $e \in T$ such that $a e=a$, and that there exists an element $t \in T$ such that $t a=e$. Whence,

$$
e^{2}=(t a) e=t(a e)=t a=e .
$$


Since $e S=S$ and $e^{2}=e, e$ is a left unit of $S$. By Lemma 2, the graph $G(\$)$ must be connected. Q.E.D.

The distance $d(A, B)$ between two vertices $A$ and $B$ of a graph is the number of edges in a shortest path between these vertices (if such a path does not exist, we define $d(A, B)=+\infty$; of course $d(A, A)=0)$. The diameter of a graph is the supremum of $d(A, B)$, where $(A, B)$ runs over all pairs of vertices of the graph. It would be interesting to know what is the diameter of the graph of a semigroup.

ADDED IN PROOF. From the December 1968 issue of Mathematical Reviews, the author has learned that Bedřich Ponděliček, in Diameter of a graph of a semigroup (Czechoslovakian, Russian and English summaries), Casposis Pěst. Mat. 92 (1967), 206-211, also solved the Bosák problem and he further obtained that the diameter of a semigroup with more than two elements does not exceed three.

\section{REFERENCES}

1. J. Bosak, "The graphs of semigroups," in Theory of graphs and its applications, Proc. Sympos. Smolenice (June 1963), Academic Press, New York, 1965, pp. 119-125.

2. A. H. Clifford and G. B. Preston, The algebraic theory of semigroups, Vol. I, Math. Surveys, No. 7, Amer. Math. Soc., Providence, R. I., 1961.

UNIVERSITY OF SOUTH FLORIDA 